\title{
Role of reactive oxygen metabolites in experimental colitis
}

\author{
A Keshavarzian, G Morgan, S Sedghi, J H Gordon, M Doria
}

\begin{abstract}
Reactive oxygen metabolites are potent inflammatory mediators that may be involved in tissue injury in inflammatory bowel disease. To evaluate their role in inflammatory bowel disease, we investigated the effects of lowering the activities of reactive oxygen metabolites in experimental colitis induced by intracolonic administration of acetic acid in rats. Intracolonic administration of $5 \%$ acetic acid caused severe inflammation (mean (SEM) inflammatory score was $24.3(0.7)$ of a maximum score of 32$)$. Acetic acid at $2 \cdot 5 \%$ produced moderate inflammation (score $=17(1.4) v 4.0$ $(0.5)$ in control rats). This lower dose was used for subsequent experiments. Specific superoxide anion scavenger methoxypolyethylene glycol:superoxide dismutase, and reactive oxygen metabolites scavenger, sulfasalazine, significantly decreased the severity of inflammation (scores: $8(4.4)$ and $9.8(2.2)$ respectively). The xanthine oxidase inhibitors, tungsten and pterin aldehyde, failed to improve inflammation but another xanthine oxidase inhibitor, allopurinol, a compound with known superoxide anion scavenging effect, did limit the inflammation (10(2)). Inhibition of hydroxyl radical production by deferoxamine or lowering hydroxyl radical values by a scavenger, dimethyl sulfoxide, did not affect the severity of inflammation. These data suggest: (1) that reactive oxygen metabolites play an important role in experimental colitis, (2) that the xanthine oxidase pathway is not a major source of reactive oxygen metabolites in colitis, and (3) that tissue injury in experimental colitis is not caused by generation of hydroxyl radicals.
\end{abstract}

Chronic idiopathic inflammatory bowel disease is a common inflammatory disorder of unknown aetiology. ${ }^{12}$ Even though the initiating factors are not known, it is clear that once the inflammation is established the typical course of the disease is such that a series of episodic acute attacks become superimposed upon chronic disease. It is also known that inflammatory mediators modulate the course of inflammatory bowel disease and play an important role during acute attacks. Among these mediators are prostaglandins and reactive oxygen metabolites. Although the role of prostaglandins has been extensively investigated, ${ }^{2-5}$ the part played by reactive oxygen metabolites has not yet been evaluated. It is known that gut is capable of generating reactive oxygen metabolites and that these seem to be important in inflammation associated with reperfusion injury after intestinal ischaemia..$^{6-10}$ Hence, it is logical to postulate that reactive oxygen metabolites may also play an important role in tissue injury in inflammatory bowel disease. We hypothesise that excessive production of reactive oxygen metabolites is a final common pathway that causes or contributes considerably to the tissue injury that occurs during acute attacks of inflammatory bowel disease. Thus, agents that lower amounts of reactive oxygen metabolites (by inhibiting their production or by scavenging) will reduce the inflammation. The aim of this study was to determine whether lowering the values of reactive oxygen metabolites could reduce the tissue injury and inflammation in an animal model of colitis.

\section{Methods}

PRODUCING COLITIS IN RATS

Female Fisher rats (Harlan, Indianapolis, IN, USA) were used. Each rat was anaesthetised using $45 \mathrm{mg} / \mathrm{kg}$ pentobarbital (Butler, Alsip, IL, USA) intraperitoneally. Then a midline incision was made and the colon was isolated. The junction of the caecum and ascending colon was identified. Two $\mathrm{ml}$ of acetic acid at various concentrations $(1 \cdot 25 \%, 2 \cdot 5 \%, 3 \%, 3.75 \%$, and $5 \%)$ were injected at this point followed by $3 \mathrm{ml}$ of air through a 25 gauge needle. Two $\mathrm{ml}$ of normal saline were substituted for acetic acid in control rats. Animals were allowed to eat and drink freely throughout the experiments. The animals were sacrificed 96 hours after intracolonic injection of acetic acid. The colon was removed and fixed in formalin for histological analysis of the severity of inflammation.

HISTOLOGICAL EVALUATION OF INFLAMMATION IN THE COLON

Coded slides were evaluated by one of the authors (MD) without knowledge of the groups of animals. The following eight criteria were used: vascular dilatation, oedema, epithelial cell loss, cellular mucin depletion, neutrophil infiltration, eosinophil infiltration, mononuclear cell infiltration, and fibrosis. Each criterion was scored on an ascending scale of $0-4$. The total possible score was 32 (absence of any abnormality $=0$, most severe inflammation $=32$ ).

\section{EXPERIMENTAL DESIGN}

Four experimental groups were studied: (a) control group - intracolonic injection of saline plus systemic administration of vehicle; (b) acetic acid group - intracolonic injection of acetic acid plus systemic administration of vehicle; (c) experimental group - intracolonic injection of acetic acid plus systemic administration of experimental agent; (d) experimental control 
group - intracolonic injection of saline plus systemic administration of experimental agent. Since acetic acid did not produce colitis in all rats each week, a minimum of four rats were used in the acetic acid group each week. The results of the experiments of only those weeks that have unequivocal colitis (score 10 or more) in all rats in the 'acetic acid group' were analysed.

\section{EXPERIMENTAL AGENTS}

The following experimental agents were used: (1) A superoxide anion scavenger, methoxypolyethylene glycol:superoxide dismutase was used (2000-5000 U/mg protein (Sigma Chemical Co St Louis, MO, USA)). Some 15000 U/kg/day were given intraperitoneally beginning on the day of intracolonic administration of acetic acid and continued daily until the rats were sacrificed. Controls rats received equal volumes of methoxypolyethylene glycol 5000 (Sigma) intraperitoneally, beginning the day of intracolonic administration of acetic acid and continued daily until the rats were sacrificed. (2) Sulfasalazine (Pharmacia, Piscataway, NJ, USA) $200 \mathrm{mg} / \mathrm{kg}$, was given orally daily for four days before the induction of colitis and continued daily until the rats were sacrificed. (3) Xanthine oxidase inhibition: three known xanthine oxidase inhibitors were given - allopurinol, tungsten, and pterin aldehyde. Each agent was started one week before the induction of colitis and was continued daily until the animals were sacrificed. The allopurinol (Sigma Chemical Co) was initially dissolved in water and titrated to $\mathrm{pH} 11$ with $\mathrm{NaOH}$ to achieve optimal dissolution. Nine rats were initially given intraperitoneal allopurinol $100 \mathrm{~kg} / \mathrm{kg}$. Because of unacceptable high mortality secondary to chemical peritonitis, however, allopurinol was given orally in a subsequent group of eight rats. The route of administration of allopurinol had no effect on the outcome and the results were subsequently pooled for analysis. Tungsten (Aldrich, Milwaukee, WI, USA), 100 $\mu \mathrm{g} / \mathrm{ml}$ of drinking water with sucrose $10 \mathrm{~g} / \mathrm{l}$, was given daily. Mean daily water consumption was $26 \mathrm{ml}$ (range: 13-27 ml). Each animal consumed a low molybdenum diet (NCI Biochemicals, Columbus, OH, USA) throughout the experiment. Commercially prepared folic acid which is contaminated with pterin aldehyde (Aldrich, Milwaukee, WI) $100 \mu \mathrm{mol} / 1$ of drinking water was given daily. Mean daily water consumption was $20 \mathrm{ml}$ (range: $18-22 \mathrm{ml}$ ). Additionally each rat received $50 \mathrm{mg} / \mathrm{kg}$ of folic acid by intragastric gavage. (4) Lowering the values of hydroxyl radicals: (a) Dimethyl sulfoxide (Sigma) was dissolved in drinking water in a 5\% concentration. Each rat was given dimethyl sulfoxide for seven days before the induction of colitis and this was continued until the animals were sacrificed. The mean daily water consumption was $31 \mathrm{ml}$ (range: $22-45 \mathrm{ml}$ ). (b) Defernxamine (Aldrich), $50 \mathrm{mg} / \mathrm{kg}$, was given intramuscularly
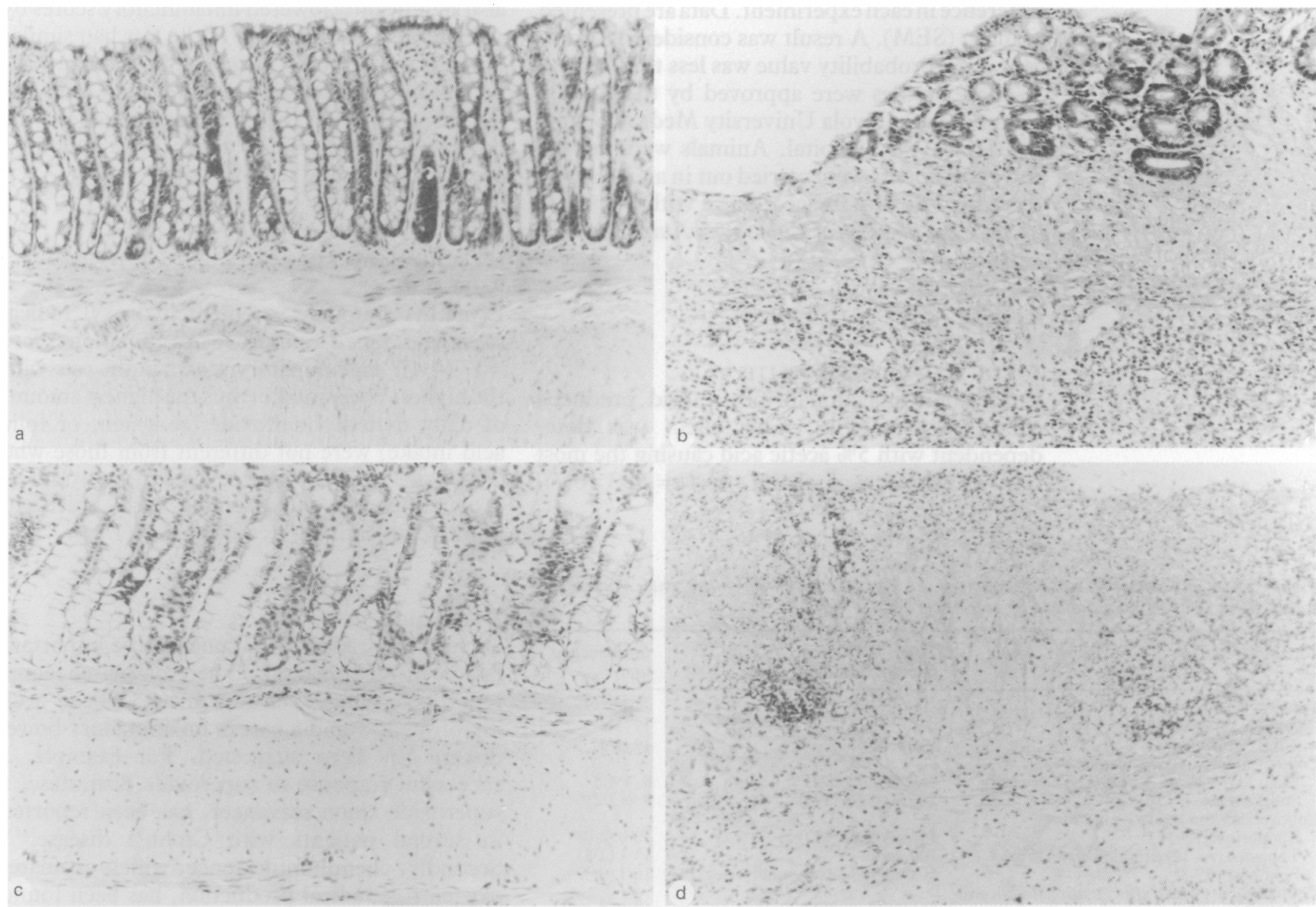

Morphological change in rat colons after intraluminal administration of acetic acid. (a) normal colon; (b) colon at 96 hours after $2 \cdot 5 \%$ acetic acid: nontransmural inflammation characterised by necrosis, ulceration, predominantly neutrophilic infiltration, and mucin depletion (inflammatory score $=16) ;(c)$ colon at 96 hours after $2.5 \%$ acetic acid and intraperitoneal allopurinol $100 \mathrm{mg} / \mathrm{kg} /$ day: significant reduction in the degree of inflammation (inflammatory score =8); (d) colon at 96 hours after $5.0 \%$ acetic acid: transmural inflammation with extensive necrosis, ulceration, and white blood cell infiltration (inflammatory score $=25$ ). (All original magnification $\times 400$. 
seven days before induction of colitis and was continued daily until animals were sacrificed.

\section{MEASUREMENT OF XANTHINE OXIDASE}

Xanthine oxidase activity was measured in colonic mucosal scrapings. Tissues were obtained from control animals and animals treated with allopurinol, tungsten, or folic acid (see treatment schedules above). Xanthine oxidase activity was measured by a standard technique." 12 In brief, colonic mucosal scrapings were suspended in 33.3 volumes (that is, $30 \mathrm{mg} / \mathrm{ml}$ ) of $50 \mathrm{mM}$ potassium phosphate buffer, $\mathrm{pH} 7 \cdot 8$, containing $1 \mathrm{mM}$ EDTA. Tissues were homogenised using a Brinkmann Polytron (Brinkmann Instruments, Westbury, NY, USA). The resulting homogenate was centrifuged for 20 minutes at 30000 $g$. The xanthine oxidase activity of the supernatant was determined spectrophotometrically by monitoring uric acid production at $295 \mathrm{nM}$. Reactions were carried out in $1.2 \mathrm{ml}$ containing $100 \mathrm{mM}$ xanthine, with or without $50 \mu \mathrm{M}$ allopurinol, at $25^{\circ} \mathrm{C}$. One $\mathrm{mU}$ of xanthine oxidase activity was defined as the amount of enzyme activity required to produce $1 \mu \mathrm{mol}$ of urate/ minute at $25^{\circ} \mathrm{C}$.

\section{STATISTICAL ANALYSIS}

Histological scores were compared between the groups of animals. The Mann-Whitney $U$ test was used to define a statistically significant difference in each experiment. Data are presented as mean (SEM). A result was considered significant if the probability value was less than 0.05 .

These studies were approved by the Animal Committee at Loyola University Medical School and Hines VA Hospital. Animals were housed and experiments were carried out in an AAAIAC accredited facility in accordance with the Guide for the Care and Use of Laboratory Animals.

\section{Results}

ACETIC ACID INDUCED COLITIS

Intracolonic injection of acetic acid produced colitis in rats (Fig). This effect was dosedependent with $5 \%$ acetic acid causing the most severe colitis (mean (SEM) score $=24 \cdot 3(0 \cdot 7)$

Mean (SEM) inflammatory scores in colons of rats given various modifying agents. Moderate colitis (score $16(1 \cdot 2))$ was induced by acetic acid $(2 \cdot 5-3 \%)$

\begin{tabular}{|c|c|c|}
\hline Groups & $\begin{array}{l}\text { Dose } \\
\text { (Route of modifying agent) }\end{array}$ & $\begin{array}{l}\text { Inflammatory } \\
\text { score }\end{array}$ \\
\hline \multicolumn{3}{|l|}{ Colitis+ vehicle } \\
\hline Colitis + saline $(n=28)$ & & $17(1 \cdot 4)$ \\
\hline Colitis + PEG $(n=6)$ & $3 \mathrm{mg}(\mathrm{IP})$ & $19(1 \cdot 4)$ \\
\hline \multicolumn{3}{|l|}{ Colitis + superoxide scavengers: } \\
\hline PEG: SOD $(n=4)$ & $15000 \mathrm{U} / \mathrm{kg} / \mathrm{d}$ (IP) & $\begin{array}{l}8(4 \cdot 4)^{\star} \\
0.8(0 \cdot 2)^{\star}\end{array}$ \\
\hline Sulfasalazine $(n=10)$ & $200 \mathrm{mg} / \mathrm{kg} / \mathrm{d}(\mathrm{PO})$ & $9 \cdot 8(2 \cdot 2)^{\star}$ \\
\hline \multicolumn{3}{|l|}{ Colitis + xanthine oxidase inhibitors: } \\
\hline Allopurinol $(n=17)$ & $100 \mathrm{mg} / \mathrm{kg} / \mathrm{d}(\mathrm{PO})$ & $10 \cdot 0(2 \cdot 0)^{\star}$ \\
\hline Tungsten $(n=12)$ drinking water $(P O)$ & $100 \mu \mathrm{g} / \mathrm{ml}$ & $15 \cdot 1(1 \cdot 8)$ \\
\hline Folic acid $(n=8)$ & $50 \mathrm{mg} / \mathrm{kg} / \mathrm{d}+100 \mu \mathrm{mol} / \mathrm{l}$ water $(\mathrm{PO})$ & $16 \cdot 1(0 \cdot 35)$ \\
\hline \multicolumn{3}{|l|}{ Colitis + agents which affect hydroxyl radicals: } \\
\hline $\operatorname{DMSO}(\mathrm{n}=8)$ & $5 \%$ in water $(\mathrm{PO})$ & $17 \cdot 0(4 \cdot 4)$ \\
\hline Deferoxamine $(n=12)$ & $50 \mathrm{mg} / \mathrm{kg} / \mathrm{d}(\mathrm{IM})$ & $21 \cdot 9(1 \cdot 1)$ \\
\hline
\end{tabular}

${ }^{\star} \mathrm{p}<0.05$ experimental group compared with control group. $\mathrm{DMSO}=$ dimethyl sulfoxide, $\mathrm{PEG}$ : $\mathrm{SOD}=$ polyethylene glycol:superoxide dismutase, $\mathrm{IP}=$ intraperitoneally, $\mathrm{PO}=$ orally, $\mathrm{IM}=$ intramuscularly. and $1 \cdot 25 \%$ failing to produce colitis $(4 \cdot 3(0 \cdot 7)$; controls: $3 \cdot 9(0 \cdot 5))$. Concentrations of $2 \cdot 5 \%$ and $3 \%$ acetic acid were used for subsequent experiments since they produced moderate colitis (score $=17 \cdot 0(1 \cdot 4)$ and $15(2 \cdot 3)$ respectively).

EFFECT OF LOWERING THE VALUES OF SUPEROXIDE ANION

A long acting, specific superoxide anion scavenger methoxypolyethylene glycol:superoxide dismutase significantly decreased the inflammation by $50 \%$ in rats (Table). Inflammatory scores in three of four rats were similar to control rats. Sulfasalazine also significantly reduced the inflammation by $40 \%$ (score $=9 \cdot 8$ $(2 \cdot 2))$. As with methoxypolyethylene glycol: superoxide dismutase, $60 \%$ of the rats had similar scores to control rats.

\section{EFFECT OF XANTHINE OXIDASE INHIBITION}

As expected, giving allopurinol, tungsten, and folic acid to the rats significantly inhibited colonic mucosal xanthine oxidase activity (control $(\mathbf{n}=$ 6): $34 \cdot 5(3 \cdot 8)$; allopurinol $(n=6): 0.47(0.47)$; tungsten $(n=6): 7.93(4 \cdot 12)$; folic acid $(n=5)$ : $14.84(5.63) \mu \mathrm{U} / \mathrm{g}$ protein). Although the percentage inhibition of experimental groups varied from $98 \%$ (alllopurinol) to $57 \%$ (folic acid), post hoc test (Scheffe's) indicated no significant differences in experimental groups. Allopurinol also significantly lowered inflammatory scores by $40 \%$ in rats to $10(2)$. Half of the rats had similar inflammatory scores to controls. In contrast, neither tungsten $($ score $=15 \cdot 1(1 \cdot 8)$ ) nor folic acid $($ score $=16 \cdot 1(0 \cdot 35))$ significantly influenced the inflammation, in spite of appreciable inhibition of xanthine oxidase activity.

EFFECT OF LOWERING THE VALUES OF HYDROXYL RADICAL

Dimethyl sulfoxide, a potent hydroxyl radical scavenger, failed to improve inflammation (score $=17(4 \cdot 4)$ ). Inflammatory scores in the rats with the highest water intake (thus the highest amount of daily dimethyl sulfoxide, tungsten, or folic acid intake) were not different from those who drank the least. Inhibition of hydroxyl radical production by deferoxamine also failed to improve inflammation $($ score $=21 \cdot 9(1 \cdot 1))$.

\section{Discussion}

Oxygen free radicals are known to be important factors in inflammation. ${ }^{13}$ Their role in ischaemia/ reperfusion injury of the gut has been well established, ${ }^{6-10}$ and a part in inflammatory bowel disease has been suggested. For example, a successful response to superoxide dismutase, a superoxide anion scavenger, has been reported in several patients with Crohn's disease. ${ }^{1+15}$ Secondly, chemiluminescence, which estimates oxygen free radical production, has been found to be raised in the white blood cells of patients with Crohn's disease. ${ }^{16}$ Finally, sulfasalazine, which is widely used in the treatment of inflammatory bowel disease, is an efficient reactive 
oxygen metabolite scavenger ${ }^{1017}$ and its therapeutic mechanism could be through its scavenging ability. Although sulfasalazine also inhibits cyclo-oxygenase (via its 5-amino salicylate component), other specific cyclooxygenase inhibitors (such as indomethacin) are not effective in the treatment of inflammatory bowel disease. ${ }^{2}$ However, direct and conclusive evidence of involvement of reactive oxygen metabolites in this disorder has been lacking.

Our present data now provide such direct evidence. These data indicate that a specific superoxide anion scavenger, superoxide dismutase, significantly improved colitis. We used methoxypolyethylene glycol:superoxide dismutase which has a similar specific activity but, unlike superoxide dismutase which has a very short half life (a few minutes), has a long half life ( 35 hours). This property makes it suitable for in vivo experiments. Moreover, we showed that sulfasalazine also significantly improves colitis. As previously discussed, this effect is, at least in part, probably due to its ability to scavenge and lower the values of reactive oxygen metabolites.

The source of superoxide anions in inflammatory bowel disease is not clear. Superoxide anions could be generated by either neutrophils which migrate to the colonic wall after the initial insult or by colonic epithelial cells. Reactive oxygen metabolites are generated intracellularly, by either NADPH oxidase in the neutrophils or by xanthine oxidase, which is present in gut mucosa. ${ }^{1318}$ Xanthine oxidase is capable of generating superoxide anions by converting hypoxanthine to xanthine and uric acid. ${ }^{6910}$ This pathway seems to be a major source of oxygen free radicals in ischaemic/reperfusion injury of the gut..$^{6-10} \mathrm{We}$ therefore evaluated the effect of three different known xanthine oxidase inhibitors - allopurinol, tungsten, and pterin aldehyde $^{1220-22}$ - and confirmed that they significantly inhibited xanthine oxidase activity in colonic mucosa. Of these three, however, only allopurinol significantly improved the severity of inflammation. Neither the tungsten nor folic acid treatment which inhibited xanthine oxidase activity improved inflammation. These findings suggest that the beneficial effect of allopurinol is a result of its known superoxide anion scavenging property. It also suggests that xanthine oxidase is not a major pathway for generation of superoxide anions in inflammatory bowel disease. A further study is required to evaluate the role of neutrophils in generation of superoxide anions in colitis.

It is known that superoxide anions cannot cause appreciable tissue injury in vivo. ${ }^{1323}$ There are two possible pathways for tissue injury caused by reactive oxygen metabolites. One, which is generally supported by in vitro studies, indicates that superoxide anions initially need to be converted to hydroxyl radicals (via the Haber Weiss reaction requiring iron), which in turn results in tissue injury. ${ }^{93} \mathrm{We}$ therefore evaluated the effects of a potent hydroxyl radical scavenger, dimethyl sulfoxide, on inflammation. Dimethyl sulfoxide had no significant effect on colitis. Furthermore, an iron chelating agent, deferoxamine, at doses that are known to inhibit hydroxyl radical production, ${ }^{10}$ did not influence the inflammation. These findings suggest that hydroxyl radicals do not play a part in tissue injury in colitis. Our finding agrees with the recent view that hypochlorous acid rather than hydroxyl radicals are involved in reactive oxygen metabolites-mediated tissue injury. ${ }^{13}$ This view is also supported by the findings of Aruoma et $\mathrm{l}^{17}$ who showed that only 5-aminosalicylic acid (an active moiety of sulfasalazine) was a hypochlorous acid scavenger. In contrast, sulfapyridine (an inactive moiety of sulfasalazine) was a more potent hydroxyl radical scavenger than either sulfasalazine or 5-aminosalicylic acid. Based on these data, they also suggested that hypochlorous acid mediates tissue damage in ulcerative colitis and that the therapeutic efficacy of sulfasalazine is due to its hypochlorous scavenging ability. Further studies are required to evaluate the mechanism of tissue injury and inflammation in the colon by reactive oxygen metabolites.

Our study strongly suggests that reactive oxygen metabolites are important in acetic acidinduced colitis in rats. Their role in human colitis needs further study. However, acetic acid induced colitis in rats shares several similarities with human ulcerative colitis. ${ }^{2+25}$ In particular, the role of prostaglandins in this animal model is very similar to ulcerative colitis, suggesting that the role of reactive oxygen metabolites may also be similar.

1 Kirsner JB, Shorter RG. Recent development in "nonspecific" inflammatory bowel disease. $N$ Engl f Med 1982 306: 775-84, 837-48.

2 McDonaldson RM. In: Sleisenger M, ed. Gastrointestinal disease. Philadelphia: WB Saunders, 1989: 1327-58, 1435-77.

3 Sharon P, Ligumsky M, Rachmilewitz D, Zor U. Role of prostaglandins in ulcerative colitis. Enhanced production during active disease and inhibition by sulfasalazine. Gastroenterology 1978; 75: 638-40.

4 Sharon P, Stenson WF. Enhanced synthesis of leukotrience B by colonic mucosa in inflammatory bowel disease. Gastroenterology 1984; 86: 453-60.

5 Boughton-Smith NK, Hawkey CJ, Whittle BJR. Biosynthesis of lipoxygenase and cyclooxygenase products from $\left[{ }^{1+} \mathrm{C}\right]$ arachidonic acid by human colonic mucosa. Gut 1983; 24 : $1175-82$.

6 Parks D, Buckley G, Granger N. Role of oxygen-derived free radicals in digestive tract disease. Surgery 1983; 94: 415-22.

7 Granger N, Hernandez L, Grishman M. Reactive oxygen metabolites: mediators of cell injury in the digestive system. Viewpoints Dig Dis 1986; 18: 13-6.

8 Granger N, Rutili G, McCord J. Superoxide radicals in feline intestinal ischemia. Gastroenterology 1981; 81: 22-9.

9 Perry M, Wadhwa S, Parks D, Pickard W, Granger N. Role of oxygen radicals in ischemia-induced lesions in the cat stomach. Gastroenterology 1986; 90: 362-7.

10 Smith S, Grisham M, Manci E, Granger N, Kvietys P. Gastric mucosal injury in the rat. Gastroenterology 1987; 92: 950-6.

11 Crissinger KD, Girsham MB, Granger DN. Developmental biology of oxidant-producing enzymes and antioxidants in the piglet intestine. Pediatr Res 1989; 25: 612-6.

12 Granger N, McCord J, Parks D, Hollwarth M. Xanthine oxidase inhibitors attenuate ischemia-induced vascular peroxidase inhibitors attenuate ischemia-induced vascular perme: $80-4$.

13 Weiss SJ. Tissue destruction by neutrophils. $N$ Engl F Med $1989 ; 320 ; 365-76$

14 Emerit J, Loeper J, Chomette G. Superoxide dismutase in the treatment of postradiotherapeutic necrosis and of Crohn's disease. Bull Eur Physiopathol Respir 1981; 17 (Suppl): 2878.

15 Niwa Y, Somiya K, Micheison AM, Puget K. Effect of liposomal-encapsulated superoxide dismutase on active oxygen-related human disorders. A Preliminary Study. Free Radic Res Commun 1988; 1: 137-53.

16 Kitahora T, Suzuki K, Asakura H, et al. Active oxygen species generated by monocytes and polymorphonuclear cells in generated by monocytes and polymorphon
Crohn's disease. Dig Dis Sci 1988; 33: 951-5.

17 Aruoma OI, Wasil M, Halliwell B, Hoey BM, Butler J. The scavenging of oxidants by sulfasalazine and its metabolites: a scavenging of oxidants by sulfasalazine and its metabolites: a
possible contribution to their anti-inflammatory effects? possible contribution to their anti-in
Bioch Pharmacol 1987; 36: 3739-42.

18 Auscher C, Amory N, Van der Kemp P, Delbarre A. Xanthine oxidase activity in human intestines. Histochemical an radiochemical study. Adv Exp Med Biol 1979; 122: 197-201. 
19 Parks D, Granger N. Ischemia-induced vascular changes: Role of xanthine oxidase and hydroxyl radicals. Am $\mathcal{F}$ Physiol 1983; $245: 285-9$.

20 Johnson J, Rajagopalan K, Cohen H. Molecular basis of the biological function of molybdenum. $\mathcal{F}$ Biol Chem 1974; 249 . 859-66.

21 Topham RW, Walker MC, Calisch MP. Liver xanthine dehydrogenase and iron mobilization. Biochem Biophys Res Commun 1982; 109: 1240-6.

22 Lewis AS, Murphy L, McCalla C, Fleary M, Purcell S.
Inhibition of mammalian xanthine oxidase by folate compounds and amethopterin. 7 Biol Chem $1984 ; 259: 12-5$.

23 Grisham M, McCord J. Chemistry and cytotoxicity of reactive oxygen metabolites. In: Taylor A, Matalon S, Ward P, eds. Physiology of oxygen radicals. Bethesda: Am Physiol Soc 1986: $1-18$

24 MacPherson B, Pfeiffer C. Experimental colitis. Digestion 1976; 14: 424-52.

25 Sharon $\mathrm{P}$, Stenson $\mathrm{W}$. Metabolism of arachidonic acid in acetic acid colitis in rats. Gastroenterology 1985; 88: 55-63. 